\title{
Why Human React Differently to the Same Sensory Experiences: an Emotion-Cognition Interaction
}

\author{
Boshra Hatef $^{1^{*}}$, Sajad Shiri², Hedayat Sahraei ${ }^{1}$ \\ ${ }^{1}$ Neuroscience Research Center, Baqiyatallah University of Medical Sciences, Tehran, Iran \\ ${ }^{2}$ Applied Biotechnology Research Center, Baqiyatallah University of Medical Sciences, Tehran, Iran
}

\section{A BSTRACT}

Introduction: Sensory experiences could be impacted the emotional state. Perception is the process that the brain selects and interprets sensations. Each perception has two physical and emotional parts. The physical part is memorized in the hippocampus and emotional part is saved in the amygdala. New perceptual experience is mixed with rehearsal memory that saved previously. Several related perceptions in different times and spaces make a concept. The collection of concepts makes beliefs. The decision making is based on their beliefs which lead to selective attention. It can be changed during the time. The amount of reward or punishment of behaviors determines the value of beliefs. If the result of action is followed by reward, the positive mood is reinforced and the emotional circuit is activated. But the cognitive system is employed if the action is followed by a punishment or conflict. The cognitive centers inhibit the emotion in a reciprocal function. Conclusion: This study reviewed data on the regions and models of emotion-cognition interaction. It was concluded that metastable model of emotional system may be a factor to produce different emotional state according to a same sensation experimentation.

\section{Key words:}

1. Sensation

2. Perception

3. Cognition

4. Emotions

* Corresponding Author: Boshra Hatef

E-mail:boshrahatef@yahoo.com 
جرا انسان واكنش متفاوتى به تجربه هاى حسى مشابه نشان مىدهد: يك تعامل هيجان -شناخت

$$
\text { بشرى هاتف"، سجاد شيرى'، هدايت صحرايى' }
$$

'مركز تحقيقات علوم اعصاب، دانشكاه علوم يزشكى بقيهالله، تهران، ايران

'مركز تحقيقات بيوتكنولوزى كاربردى، دانشكاه علوم يزشكى بقيهاله، تهران، ايران

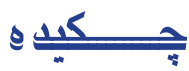

مقدمه: تجربيات حسى مى توانند وضعيت هيجانى را درگير كنند. ادراك روندى است كه مغز احساسات

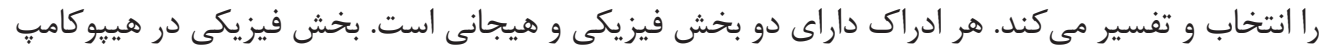

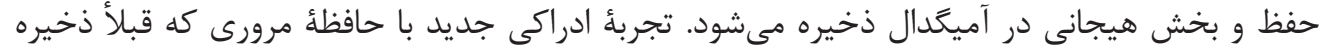

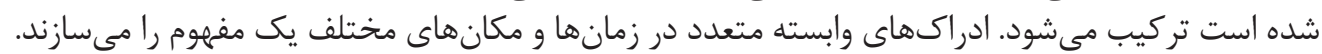

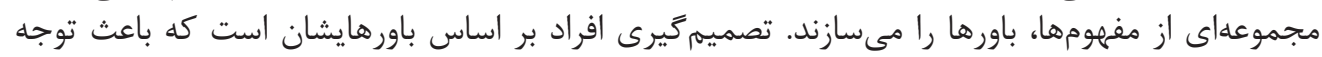

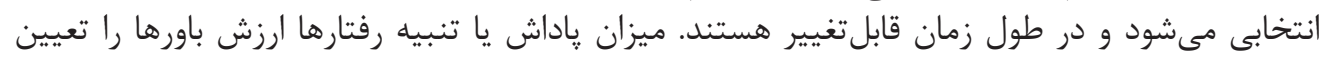

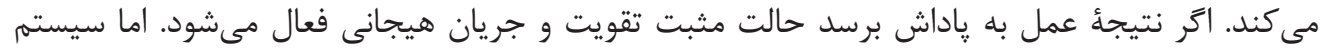

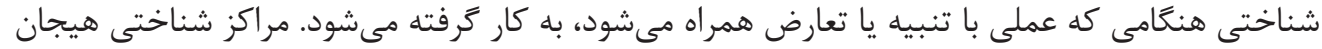

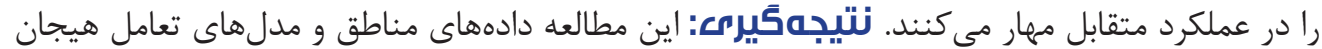

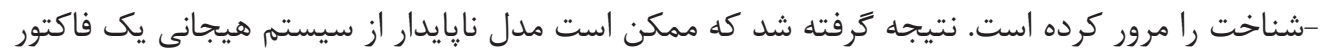

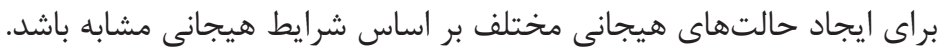

كليد وازهها:

إحساس

ז. ادراك

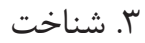

( )

" نويسنده مسئول: بشرى هاتف آدرس الكترونيكى: boshrahatef@yahoo.com 
به نوع تقويت

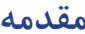

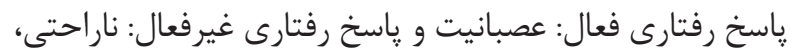

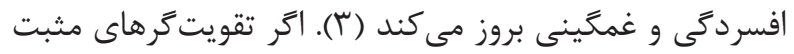

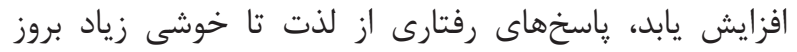

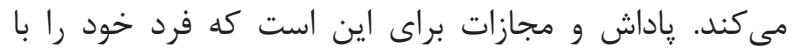

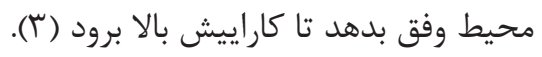

در تعريف شناخت مىتوان كَفت فرايندهاى شناختى، بخش مئر

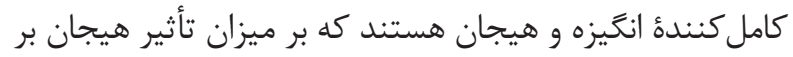

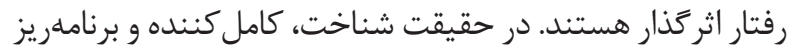

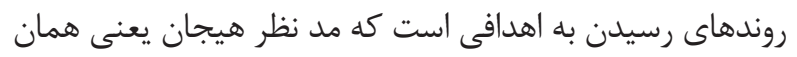

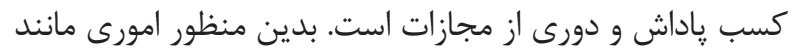

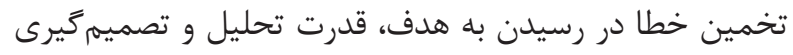

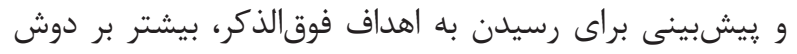

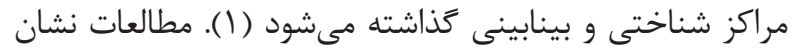

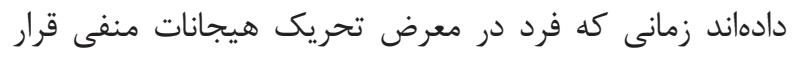

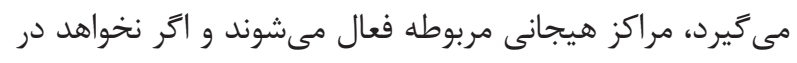

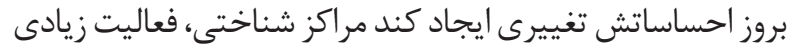

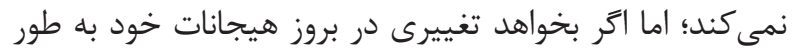

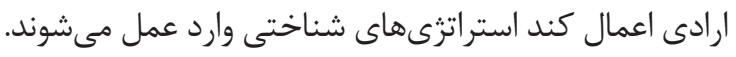

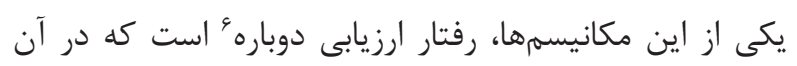

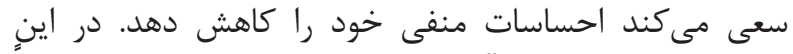

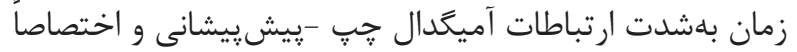

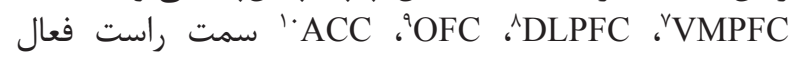

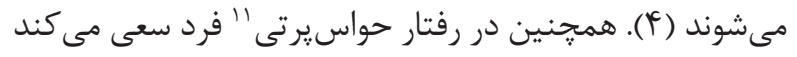

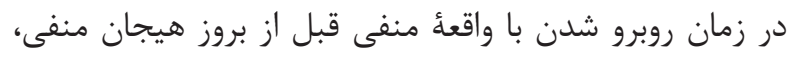

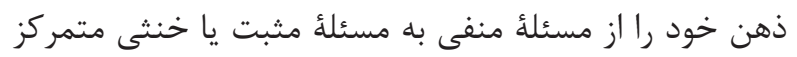

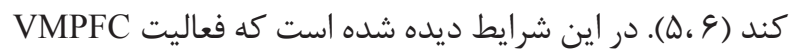

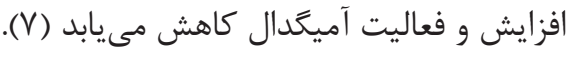

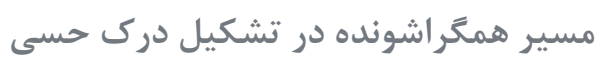

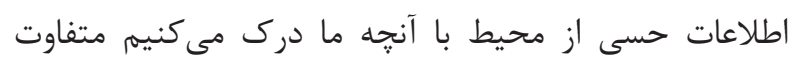

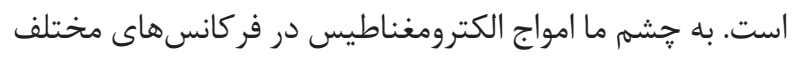

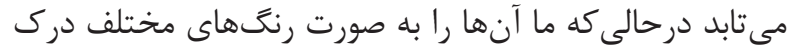

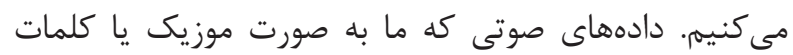

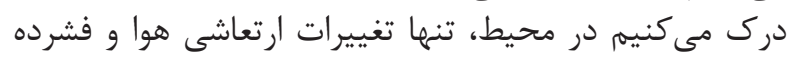

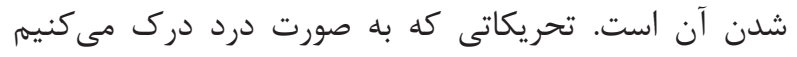

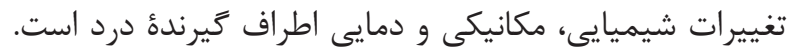

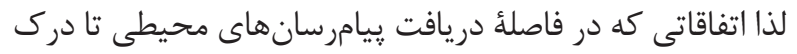

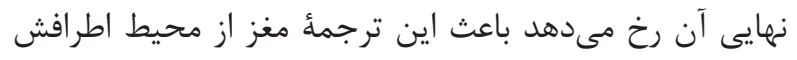

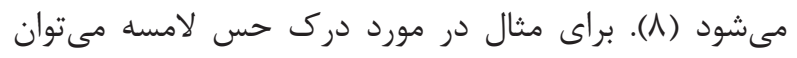

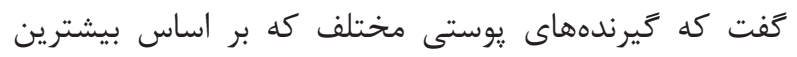

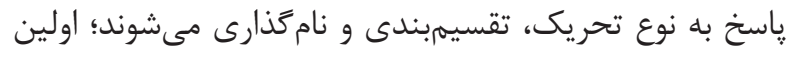

${ }^{1}$ Emotional state

${ }^{2}$ Cognition

${ }^{3}$ Motivation

${ }^{4}$ Reward

${ }^{5}$ Punishment

${ }^{6}$ Reappraisal

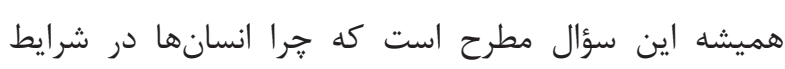

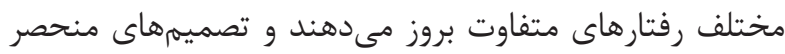

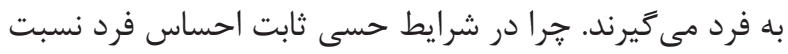

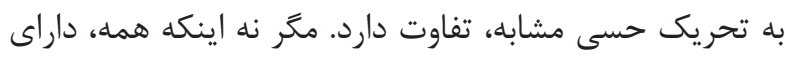

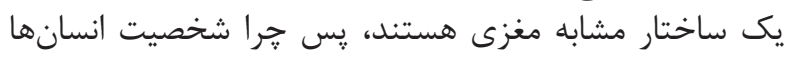

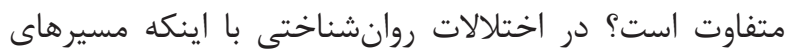

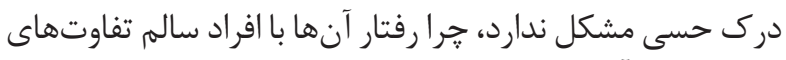

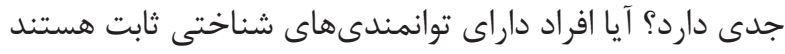

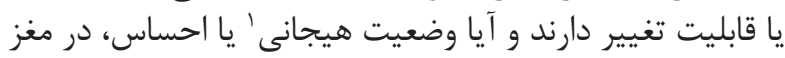

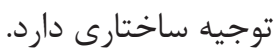

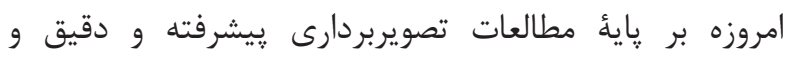

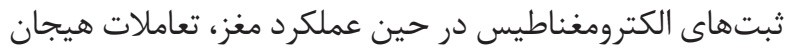

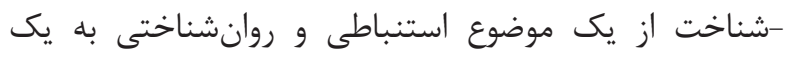

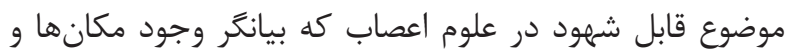

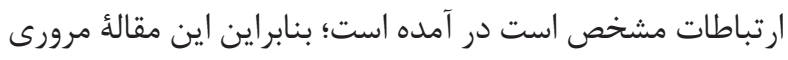

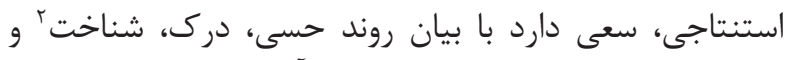

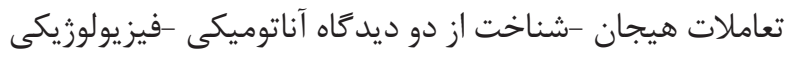
و مدلسازى به سؤالات فوق بيردازد.

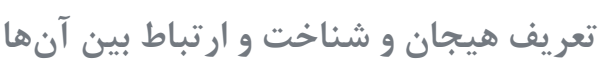

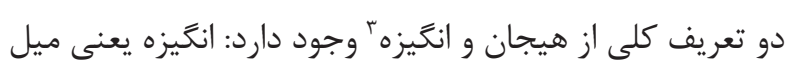

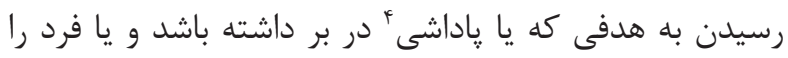

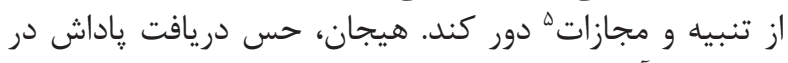

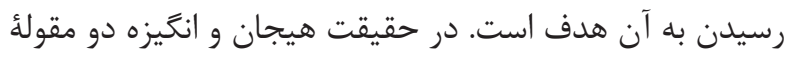

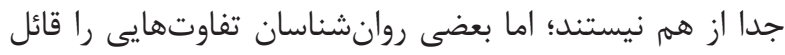

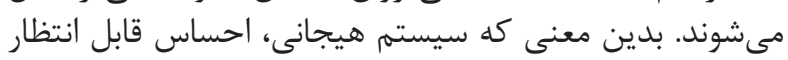

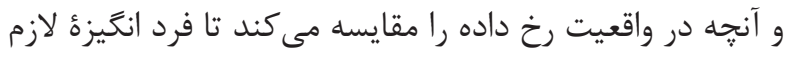

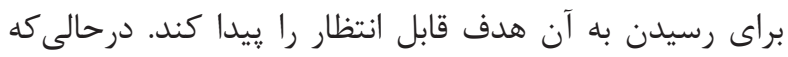

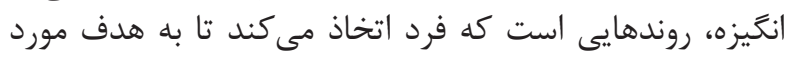

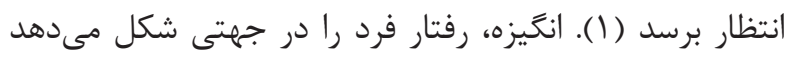

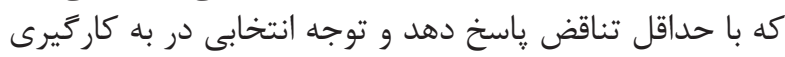

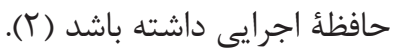

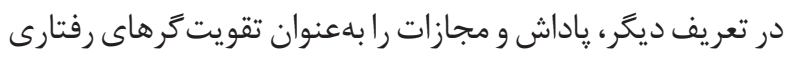

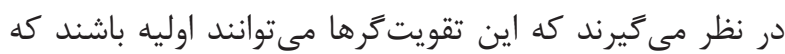

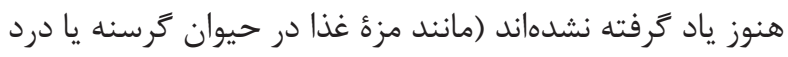

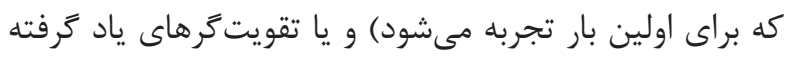

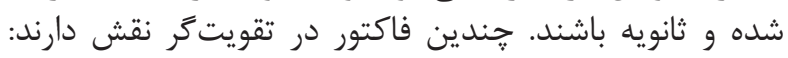

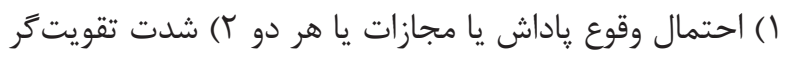

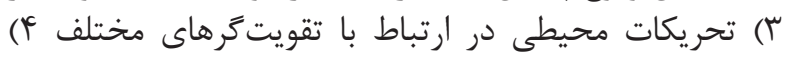

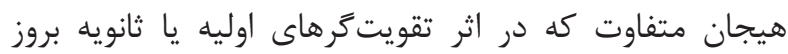

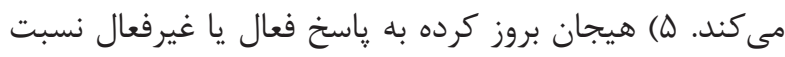

\footnotetext{
${ }^{7}$ Ventromedial prefrontal cortex

${ }^{8}$ Dorsolateral prefrontal cortex

${ }^{9}$ Orbitofrontal cortex

${ }^{10}$ Anterior cingulate cortex

${ }^{11}$ Distraction
} 
وارد و درك مىشود. در ضمن اطلاعات مناطق دورتر هم همغرا

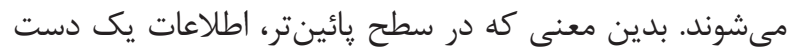

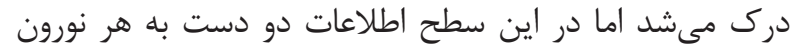

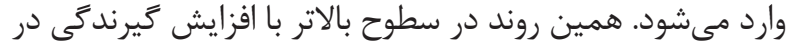

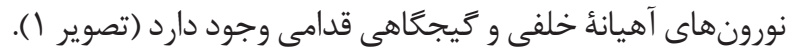

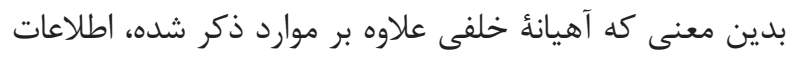

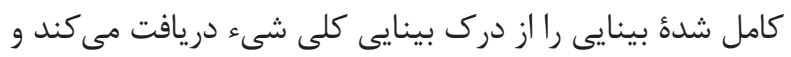

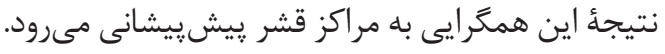

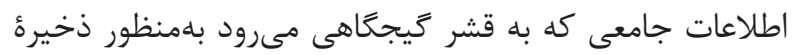

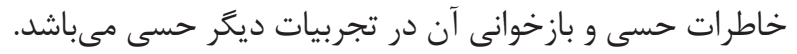

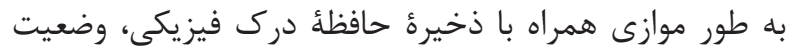

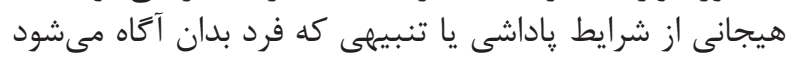

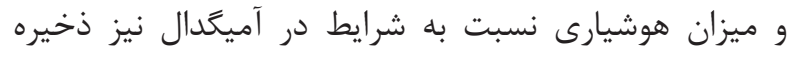
مى

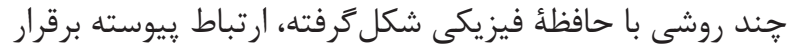

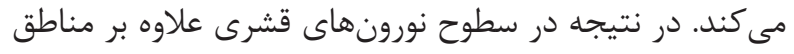

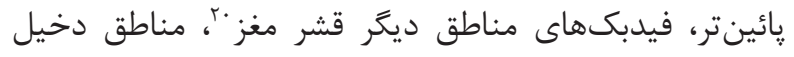

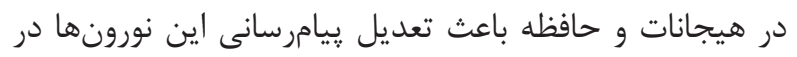

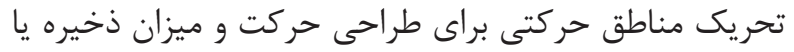

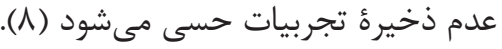

نقش سيستم ليمبيك و قشر وِيش بيشانى در تنظيم

$$
\text { حس و حركت ست ستم }
$$

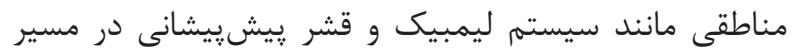

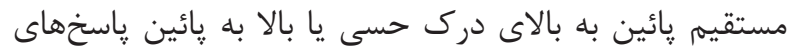

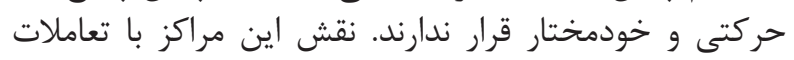

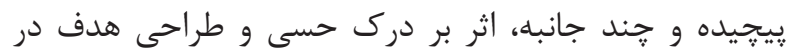

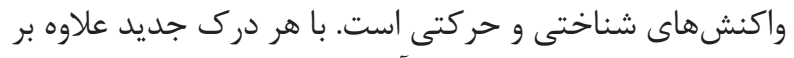

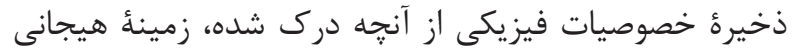

قدم در ترجمأ محيط براى مغز ما هستند. سه گروه بزرى آن آنها

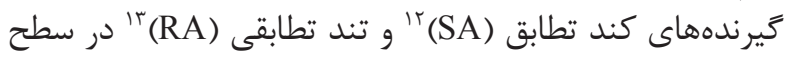

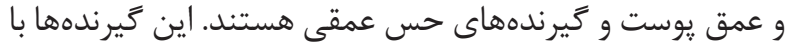

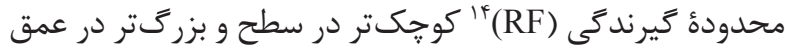

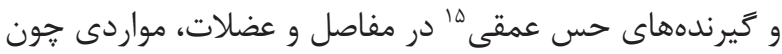

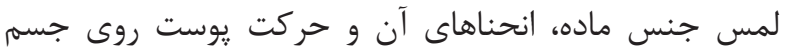

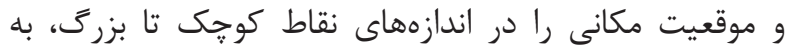
تالاموس و از آنجا به مناطق اولئ درأ حسى يعنى محدودئ قشرى 19 3a 3 و 3b مى مرستند.

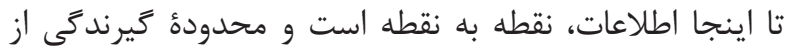

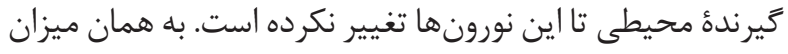

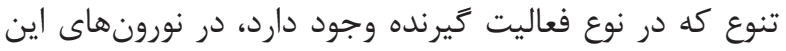

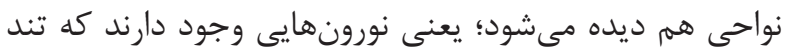

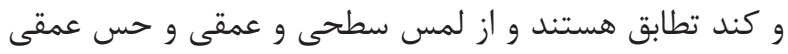

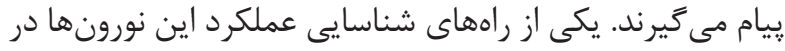

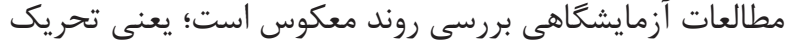

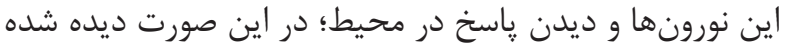

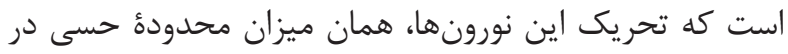

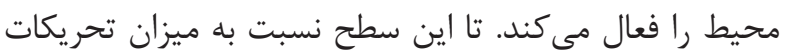

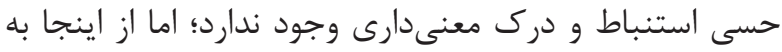

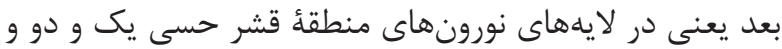

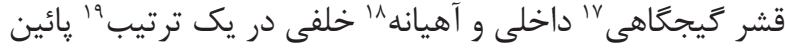

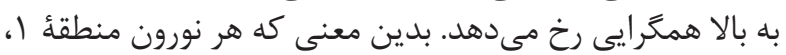

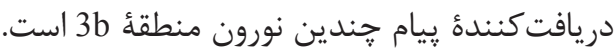

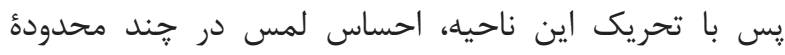
كيرند

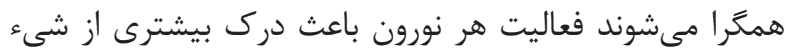

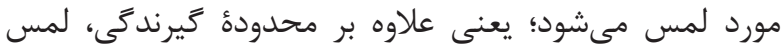

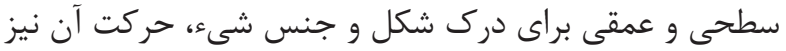

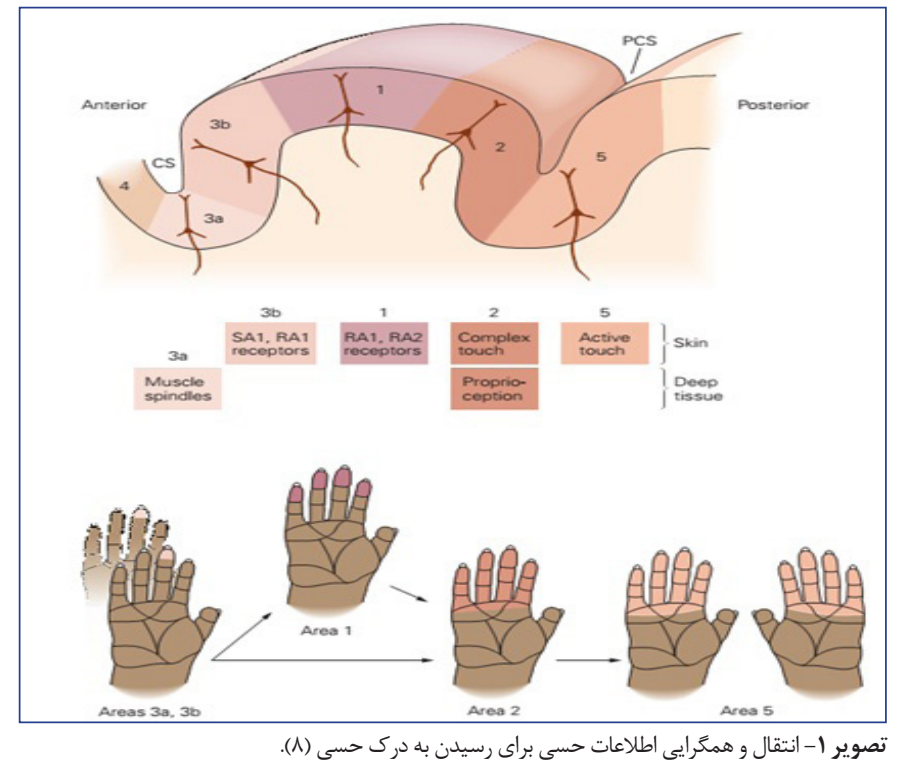

\footnotetext{
${ }^{12}$ Slowly adapting receptors

${ }^{13}$ Rapidly adapting receptors

${ }^{14}$ Receptive field

${ }^{15}$ Proprioceptive receptors

${ }^{16}$ Cortical
}
${ }^{17}$ Temporal
${ }^{18}$ Parietal
${ }^{19}$ Hierarchy
${ }^{20}$ Cortex 


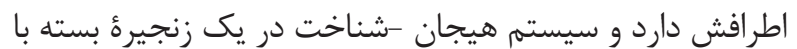

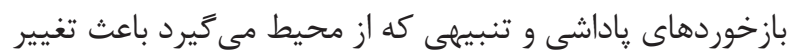

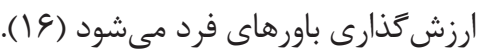

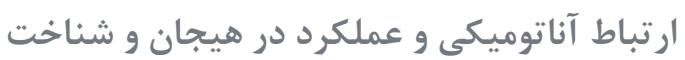

از لحاظ ساختارى ثابت شده است كه آميحدال قطب اصلى

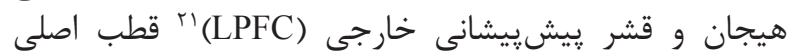

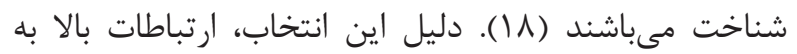

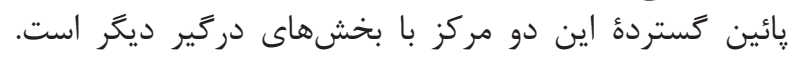

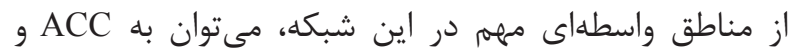

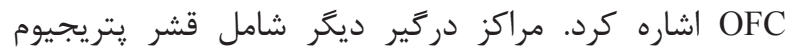

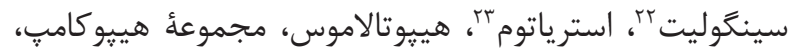

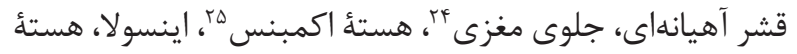

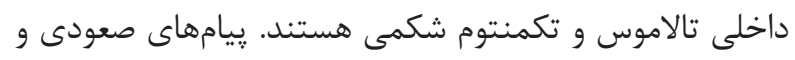

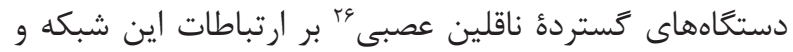

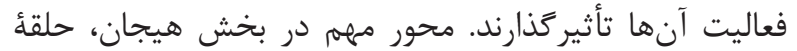

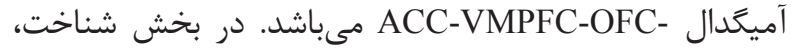

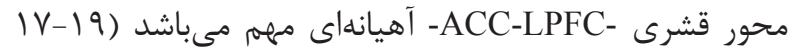

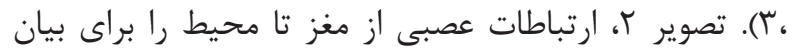
ارتباط هيجان -شناخت به تصوير كشيده است.

با توجه به ميزان تجمع زمانى و مكانى ارتباطات مناطق فوق،

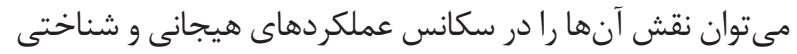

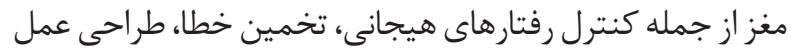

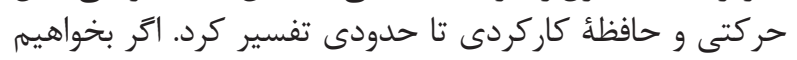

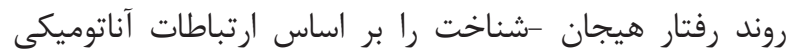

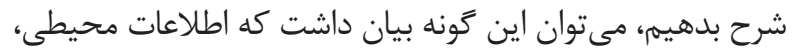

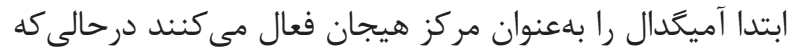

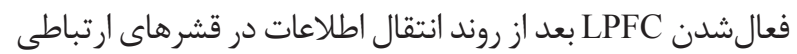

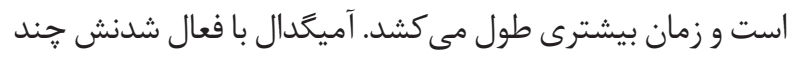

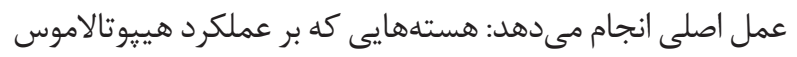

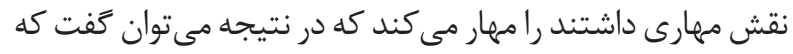

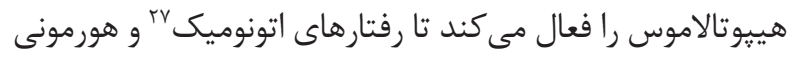
مربوط به افزايش هيجان شروع شود.

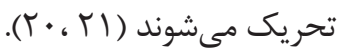

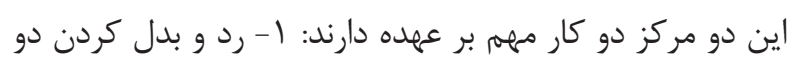

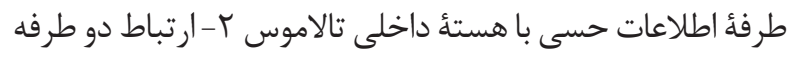

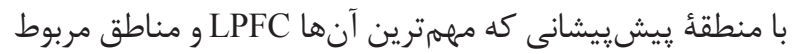

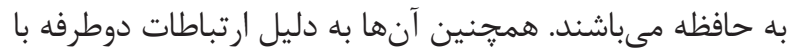

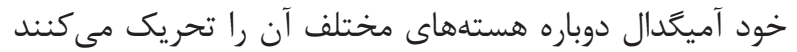

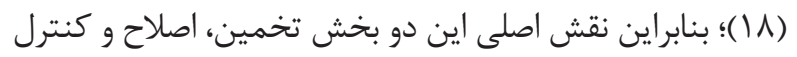

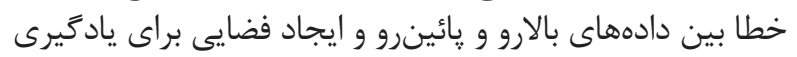

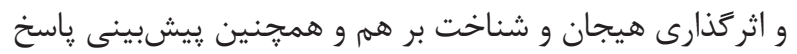

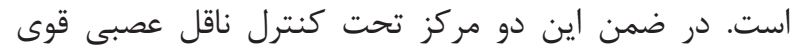

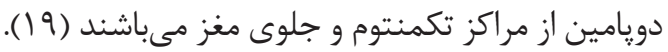

\footnotetext{
${ }^{21}$ Lateral prefrontal cortex

${ }^{22}$ Pterygium cingulate

${ }^{23}$ Striatum

${ }^{24}$ Forebrain
}

و انكيزشى مربوط به محيط اطراف مورد درك شده شدر نيز ذخيره

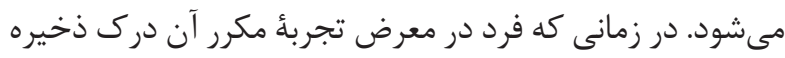

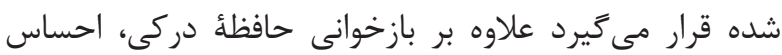

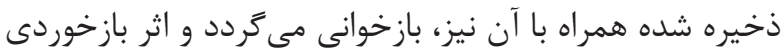

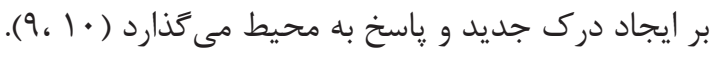

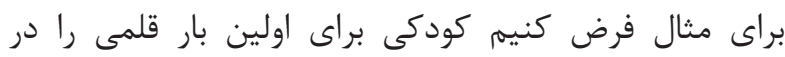

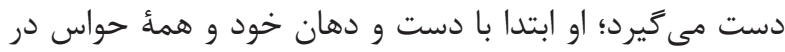

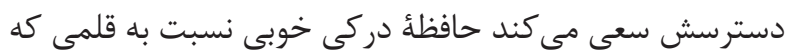

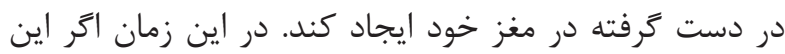

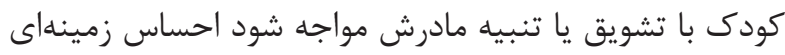

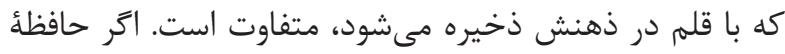

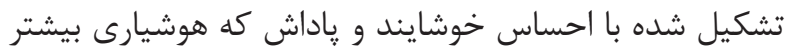

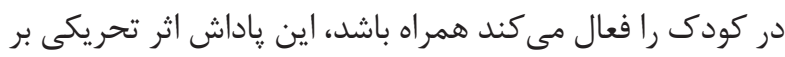

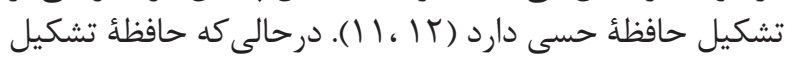

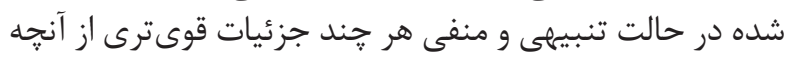

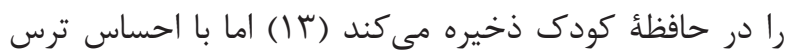

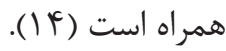

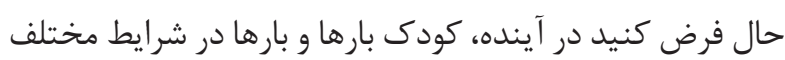

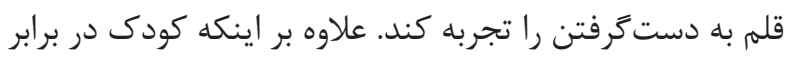

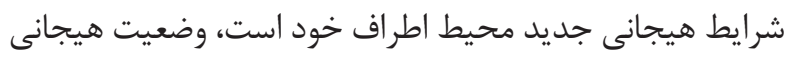

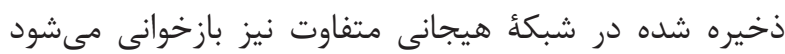

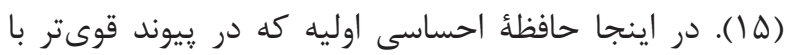

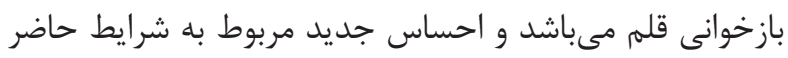

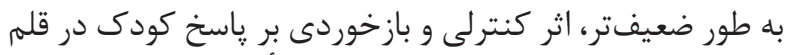

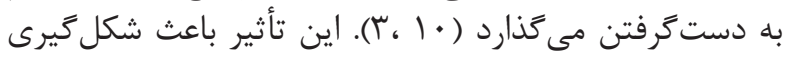

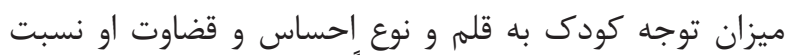

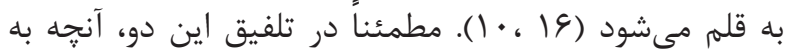

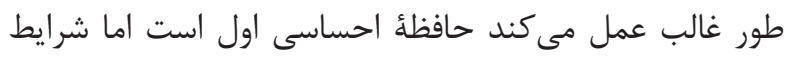

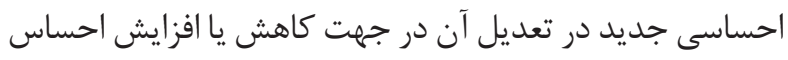

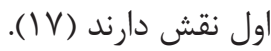

شبكؤ هيجانى كه قطب مركزى آن آميحدال است در ارتباط

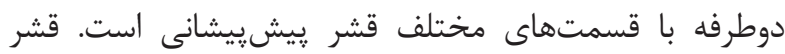

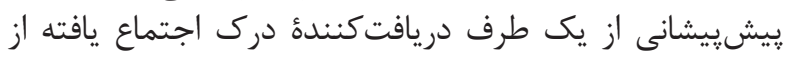

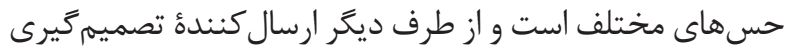

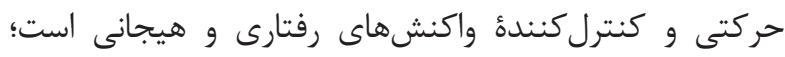

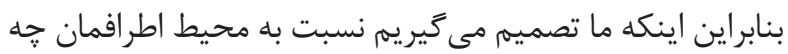

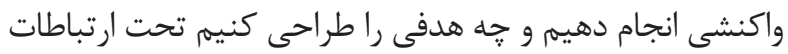

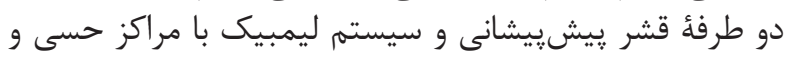

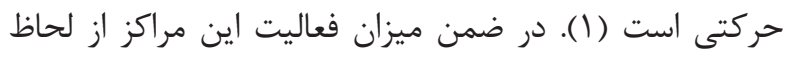

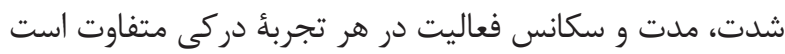

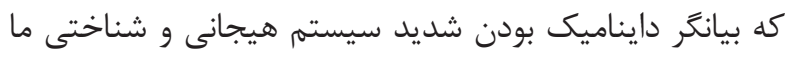

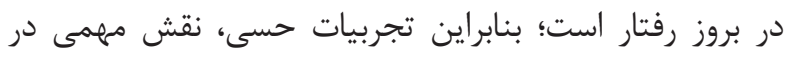

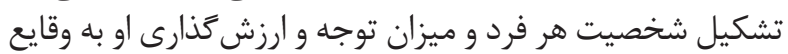

\footnotetext{
${ }^{25}$ Accumbens nucleus

${ }^{26}$ Neurotransmitter

${ }^{27}$ Autonomic
} 


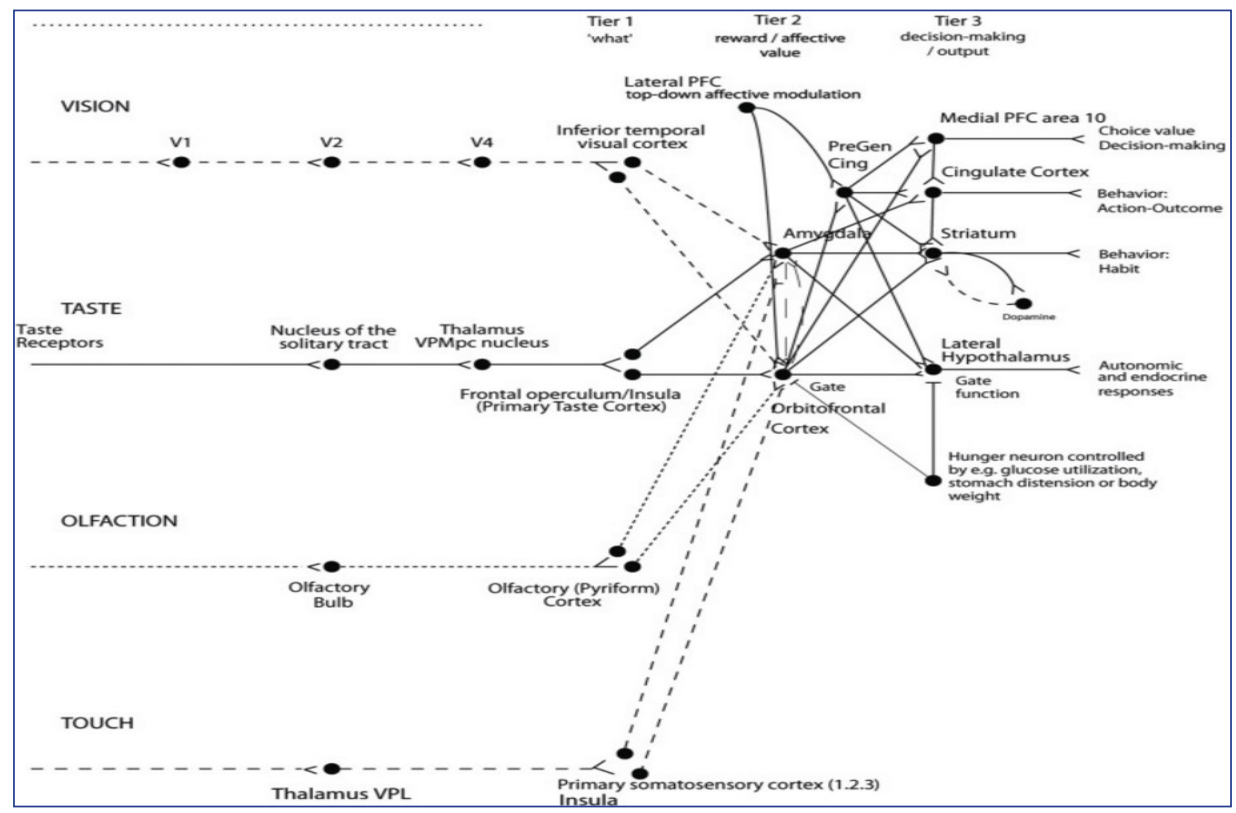

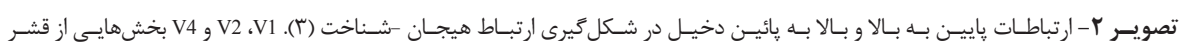

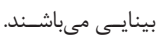

درد و حس ناخوشايند نسبت به آن ذخيره مىشود و و حافظهُ

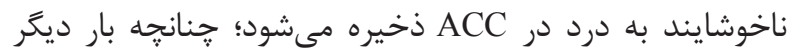

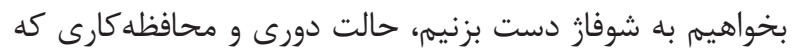

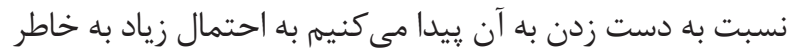

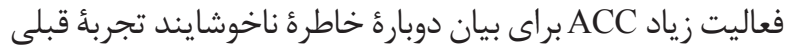

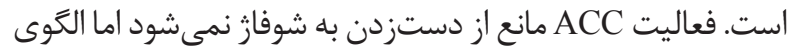

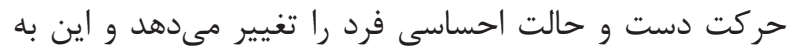

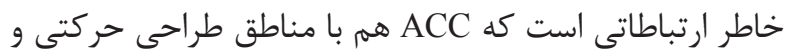

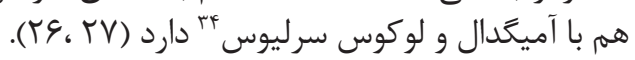

بخشهاى قدامى و شكمى ACC در بروز ياسخ خلقى درد دخيل

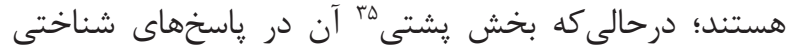

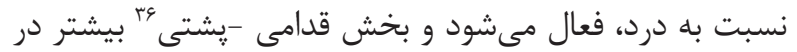

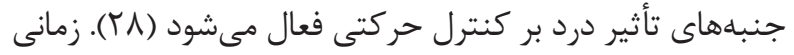

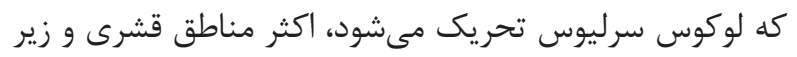

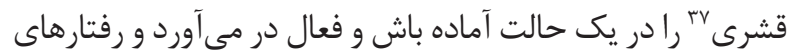

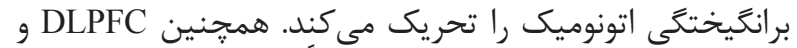

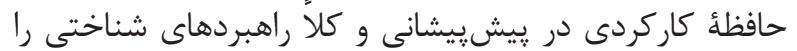

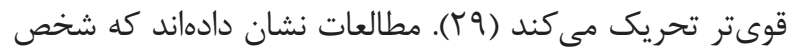

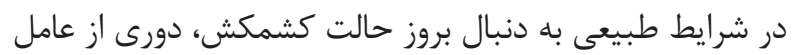

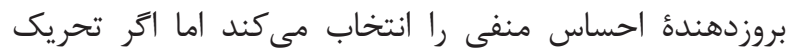

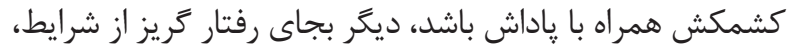

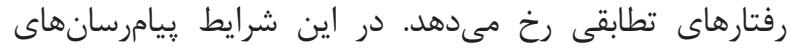

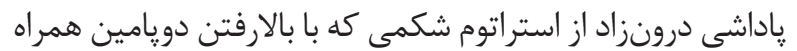

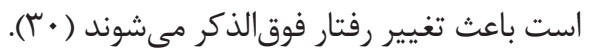

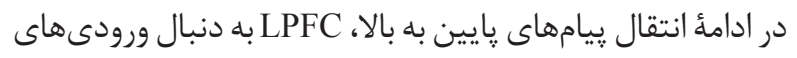

\footnotetext{
${ }^{28}$ Basolateral

${ }^{29}$ Positive value-coding

${ }^{30}$ Negative value-coding

${ }^{31}$ Long term potentiation

${ }^{32}$ Long term depression
}

بCC بيشتر، جنبههاى پِيشبينى -حركتى و OFC بيشتر

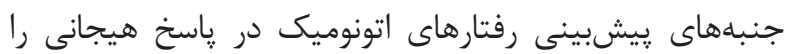

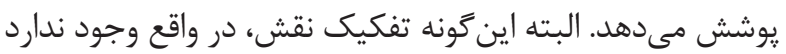

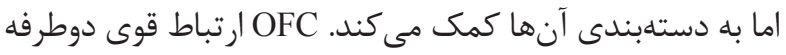

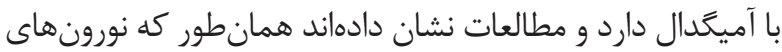

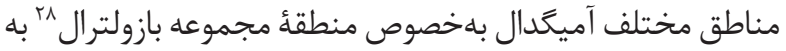

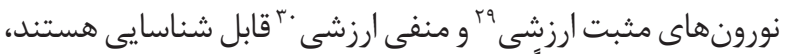

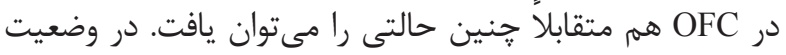

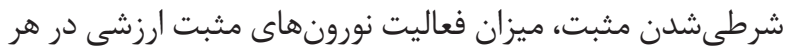

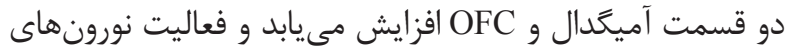
منفى ارزشى كاهش مى يابد و برعكس (IV)

OFC در ارتباطات دوطرفه با OMPFC مىباشد كه اين ناحيه

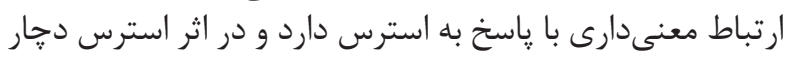

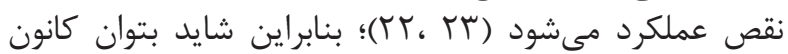

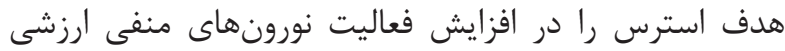

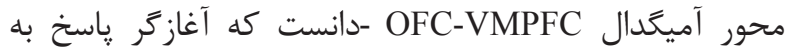

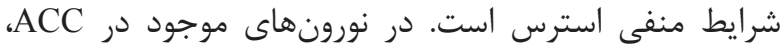

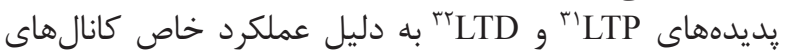

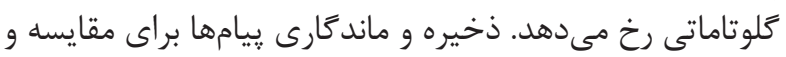

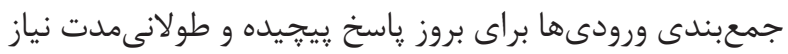

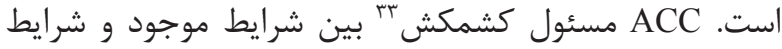

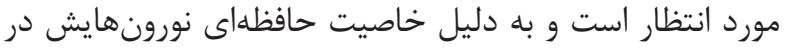
ييشبينى رفتارى نسبت به اعمالى كه در آينده انجام خواهيم

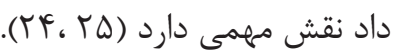
براى مثال وقتى يك بار به شوفاز داغ دست مىزنيم تحريك
${ }^{33}$ Conflict
${ }^{34}$ Locus coeruleus
${ }^{35}$ Dorsal
${ }^{36}$ Dorsocaudal
${ }^{37}$ Sub-cortical 
در عمل مقايسٔ خطا و كنترل تناقضها بين دادههاى سيستم

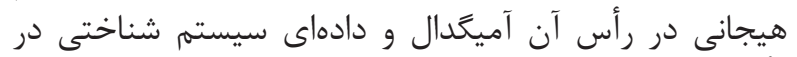

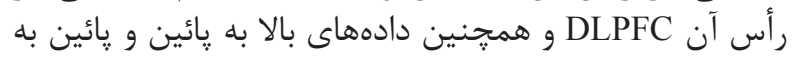

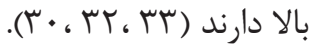

در نتاه سيستماتيك، Pessoa از لحاظ مفهومى طرحى را راطى

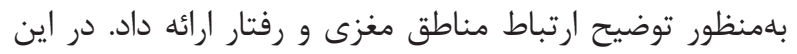

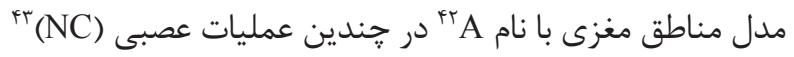

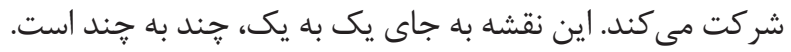

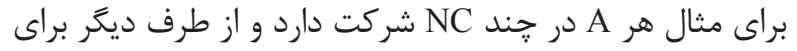

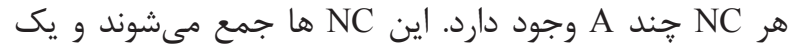

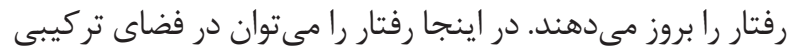

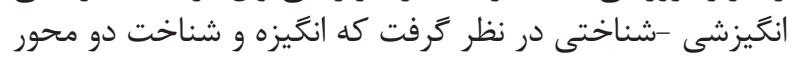

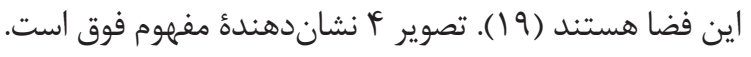

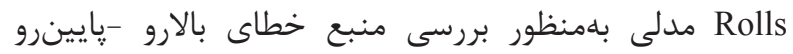

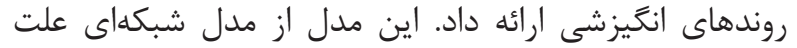

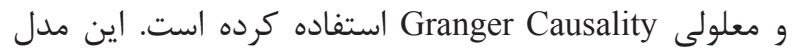

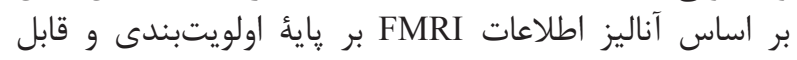

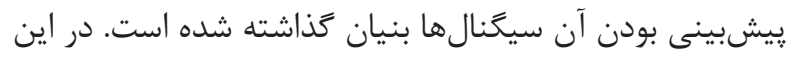

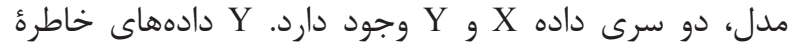

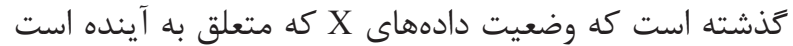

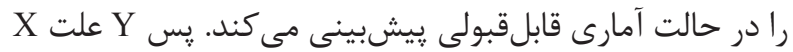

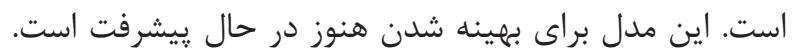

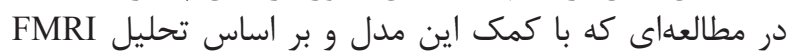
انجام شده است، نشان داده است كه تأثير بالا به يائين

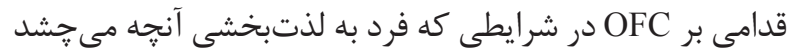

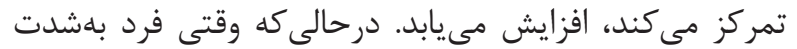

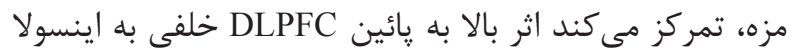

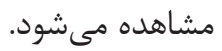

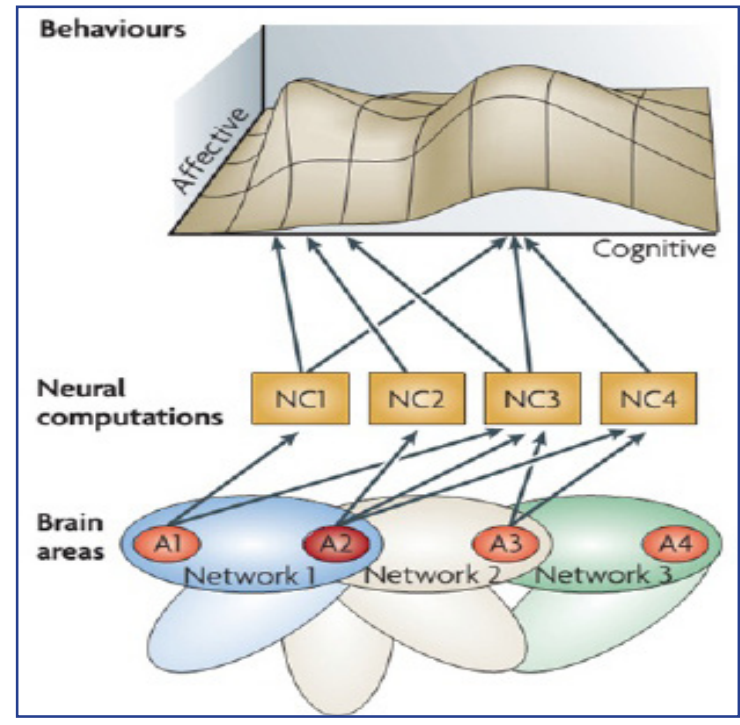

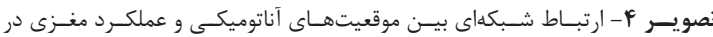

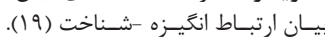

${ }^{38}$ Parietal

${ }^{39}$ Ghashghaei

${ }^{40}$ Metastable
حسى از قشرهاى ارتباطى آهيانهاى ^^و مناطق مرتبط با حافظه، و و ديخر مناطق مرتبط، فعال مىشود.

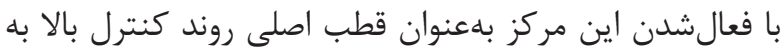

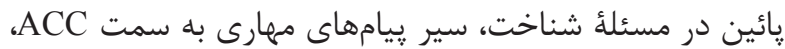
OFC و MVPFC سرازير مىشود. در نتيجه، تمام تعاملاتى مئي

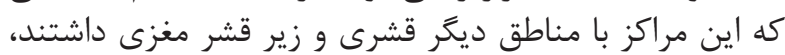

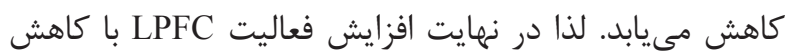

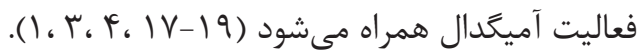
در مورد نوع عملكرد نورونهاى OFC گفته ميىشود كه فعال

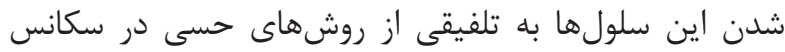

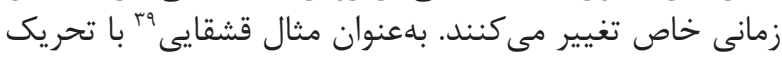

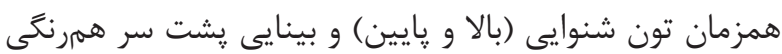

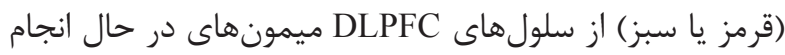

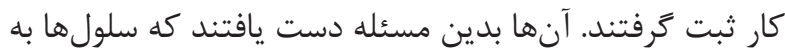

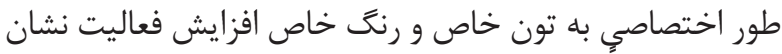

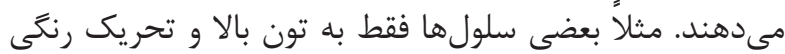

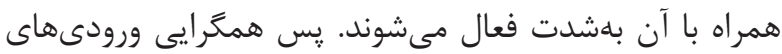

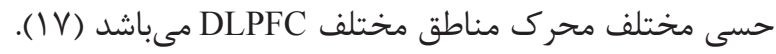

مدلهاى دايناميك در ارتباط شناخت و هيجان بر اساس مدلهاى مختلف ارائه شده در ارتباط هيجان و

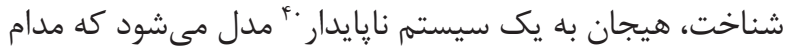

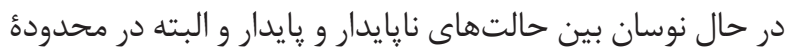

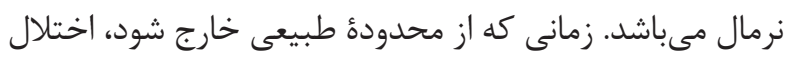

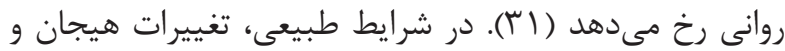

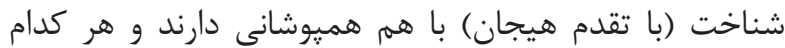

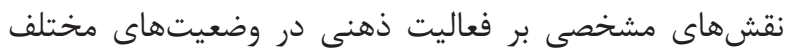

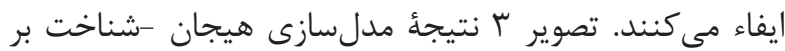

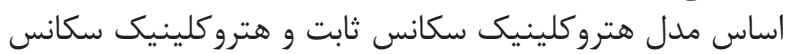

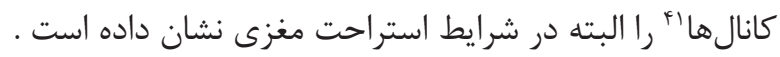
در زمانى كه هيجان در سطح بالا نوسان مىكند شناخت در

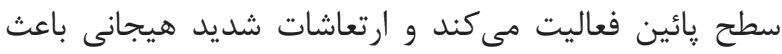
بروز مشكلات شناختى مى شود. ACC و OFC و

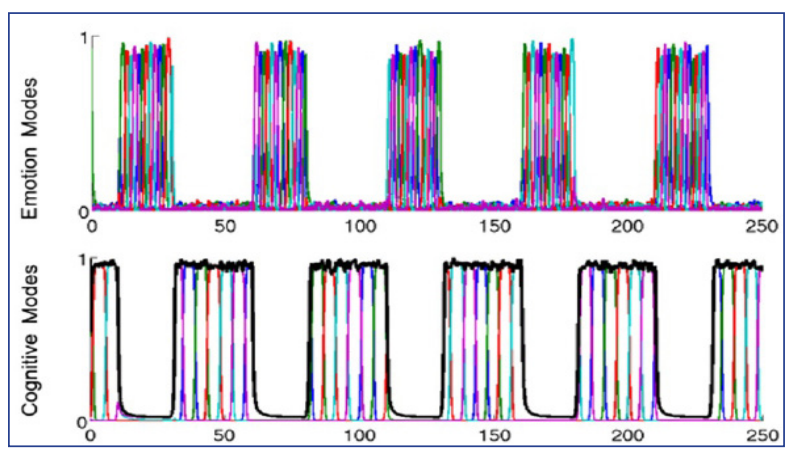

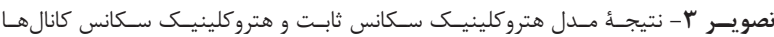

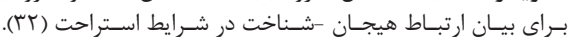

${ }^{41}$ Stable heteroclinic sequence and channels heteroclinic sequence ${ }^{42}$ Area

${ }^{43}$ Neural computation 
انخَيزشى و اثركٍذارى تحريكات است كه با هم در تصوير B ادغام

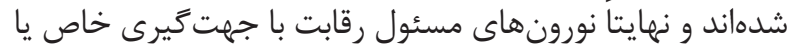

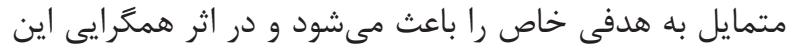

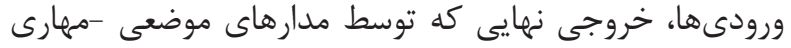

تعديل مىشود، بيرون مى آيد.

يكى از مهمترين مسيرهاى بازخوردى در تنظيم خطا و متمايل

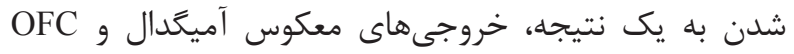

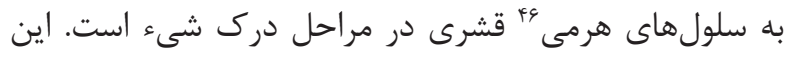

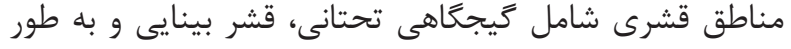

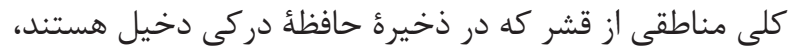

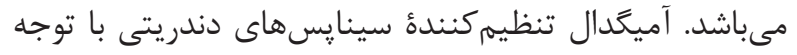

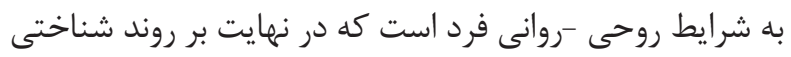

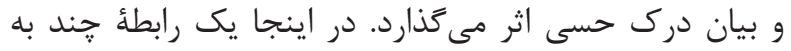

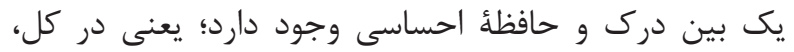

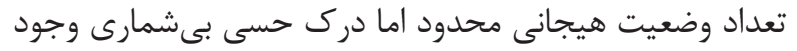

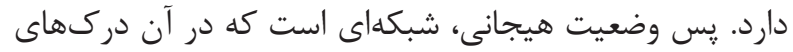

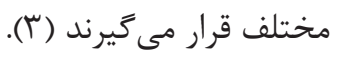

نتيجه

هدف از اين مقاله، تحليل رفتارهاى مختلف افراد به تجربيات

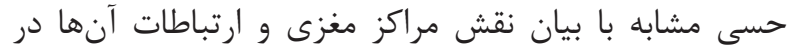

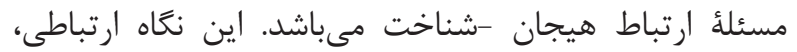

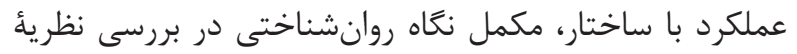

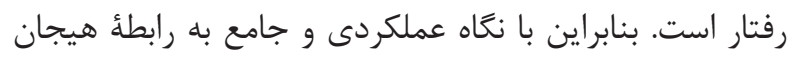

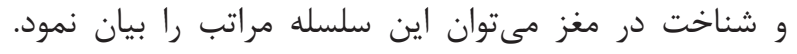

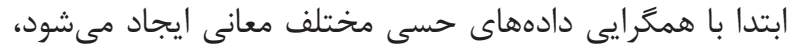

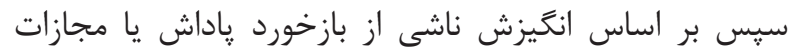

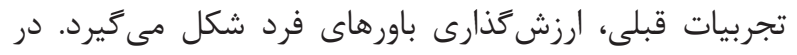

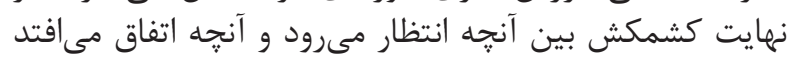

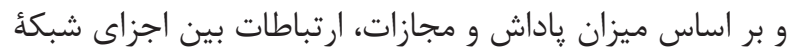

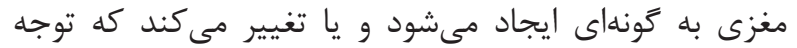

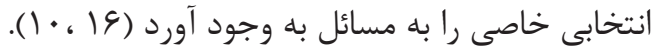

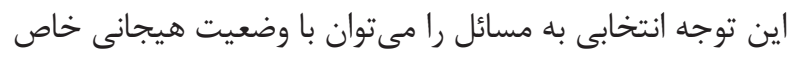

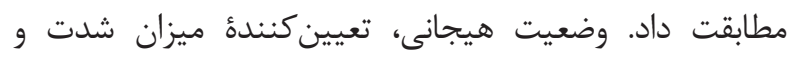

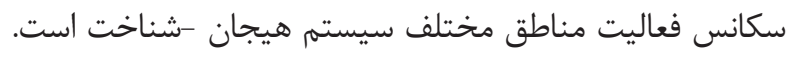

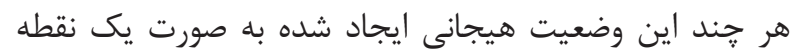

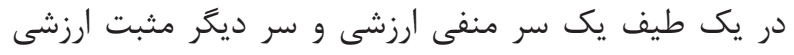

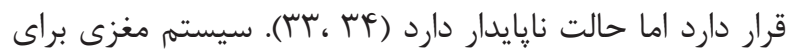

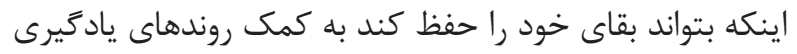

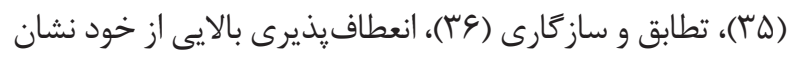

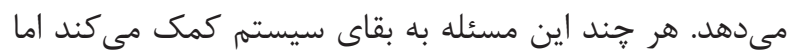

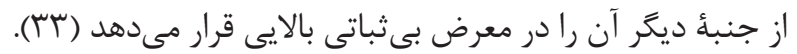

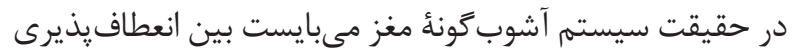

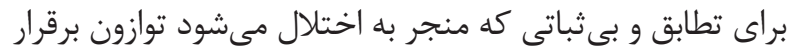

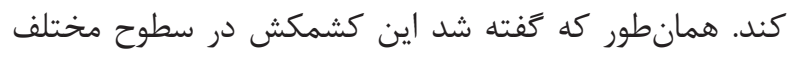

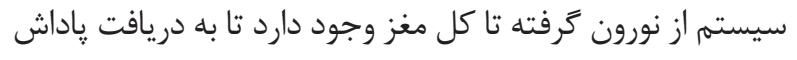

${ }^{44}$ Biased activation theory

${ }^{45}$ Local

${ }^{46}$ Pyramidal
اين نتايج را مطالعات آزمايشخاهى هم تأييد كردهاند. اين مدل

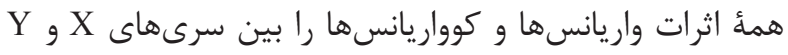

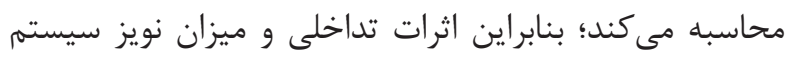

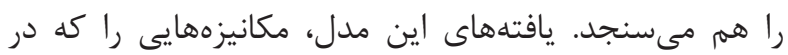

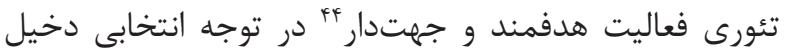

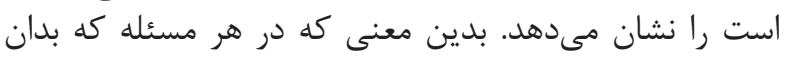

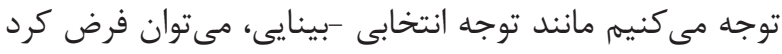

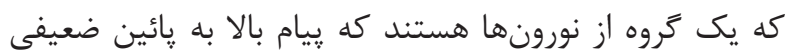

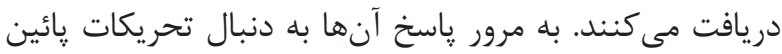

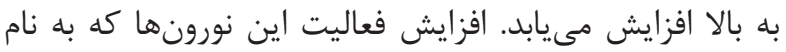

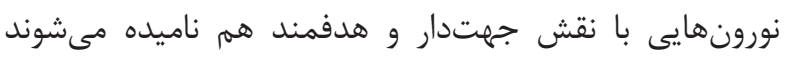

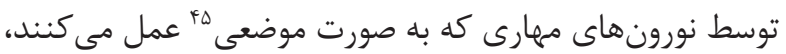

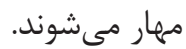

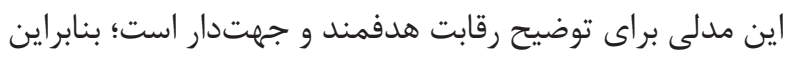

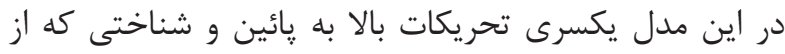

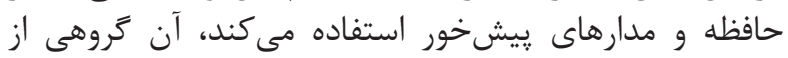

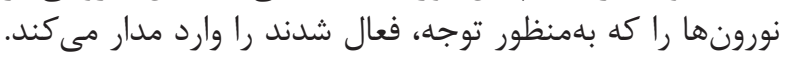

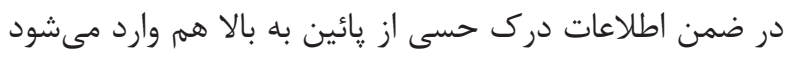

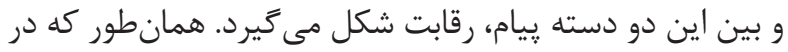

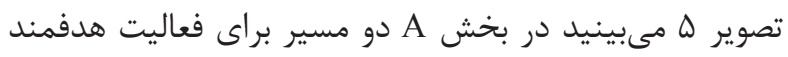

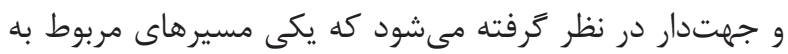

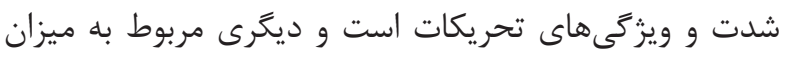

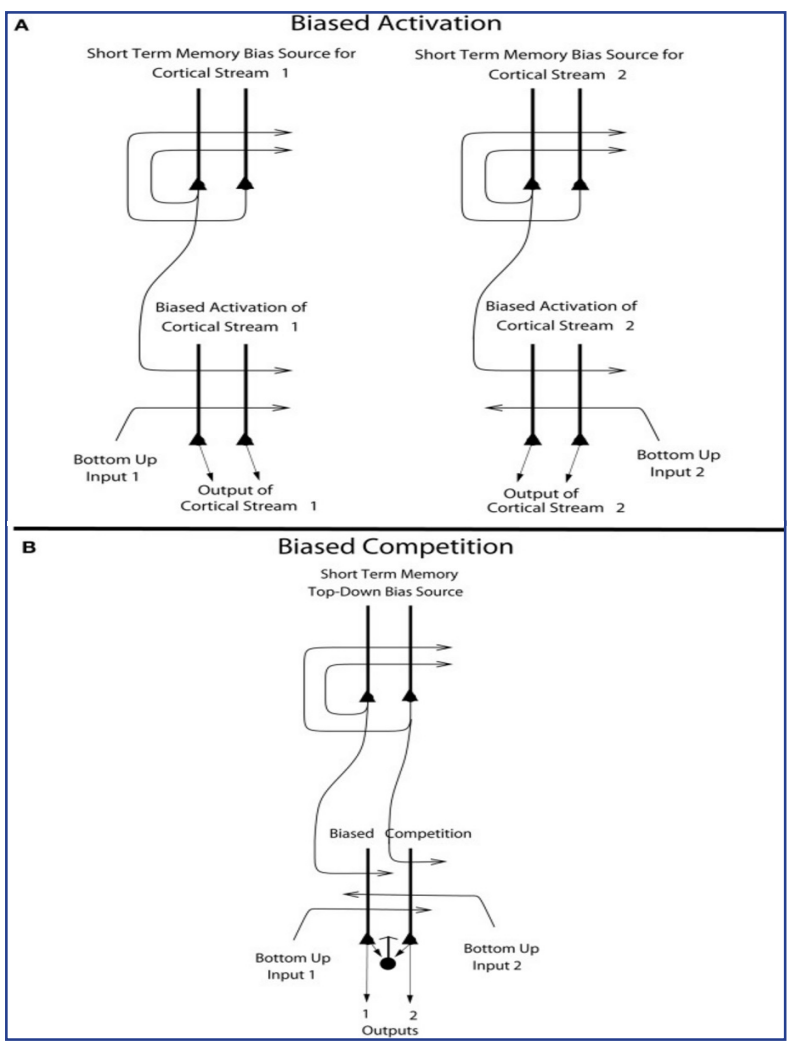

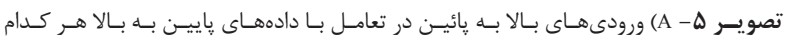

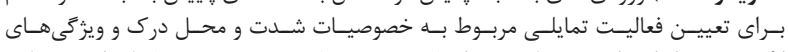

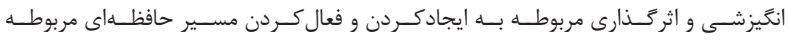

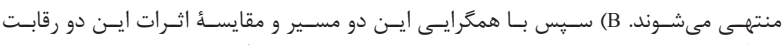

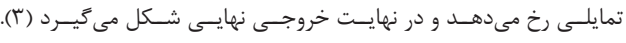




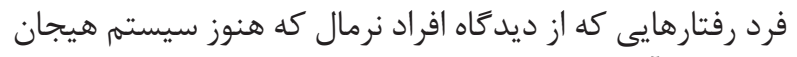

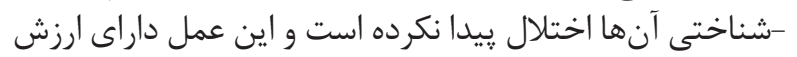

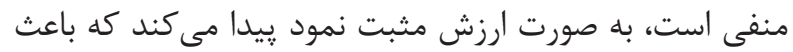

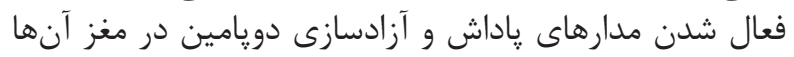

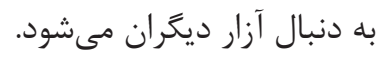

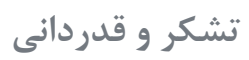
در اينجا بر خود لازم مى دانيم از اساتيد محترم جناب آقاى دكتر

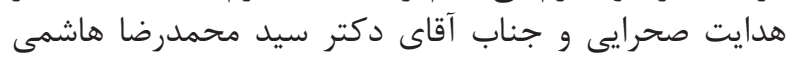

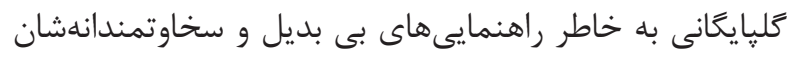
تشكر و قدردانى نماييم.

1. Crocker LD, Heller W, Warren SL, O'Hare AJ, Infantolino ZP, Miller GA. Relationships among cognition, emotion, and motivation: implications for intervention and neuroplasticity in psychopathology. Front Hum Neurosci. 2013; 7: 261. doi: 10.3389/ fnhum.2013.00261.

2. Pessoa L. Précis of the cognitive-emotional brain. Behav Brain Sci. 2015; 38: e71. doi: 10.1017/ S0140525X14000120.

3. Rolls ET. A biased activation theory of the cognitive and attentional modulation of emotion. Front Hum Neurosci. 2013; 7: 74. doi: 10.3389/fnhum.2013.00074.

4. Banks SJ, Eddy KT, Angstadt M, Nathan PJ, Phan KL. Amygdala-frontal connectivity during emotion regulation. Soc Cogn Affect Neurosci. 2007; 2(4): 303-12.

5. Tolegenova AA, Kustubayeva AM, Matthews G. Trait meta-mood, gender and EEG response during emotionregulation. Pers Individ Dif. 2014; 65(0): 75-80.

6. Gyurak A, Gross JJ, Etkin A. Explicit and implicit emotion regulation: a dual-process framework. Cogn Emot. 2011; 25(3): 400-12.

7. Moyal N, Henik A, Anholt GE. Cognitive strategies to regulate emotions-current evidence and future directions. Front Psychol. 2014; 4: 1019. doi: 10.3389/ fpsyg.2013.01019.

8. Kandel ER, Schwartz JH, Jessell TM, Siegelbaum SA, Hudspeth AJ. Principles of neural science. 5th ed. New York. McGraw-Hill Education. 2013.

9. Larkum M. A cellular mechanism for cortical associations: an organizing principle for the cerebral cortex. Trends Neurosci. 2013; 36(3): 141-51.

10. Lerner JS, Li Y, Valdesolo P, Kassam KS. Emotion and

$$
\begin{aligned}
& \text { بيشتر و مجازات كمتر منتهلى شود (V ، V ، (1). احتمال مىرود }
\end{aligned}
$$

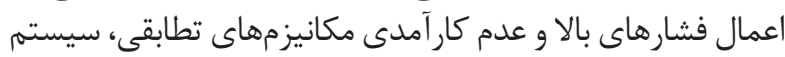

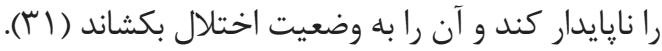

$$
\begin{aligned}
& \text { ممكن است تجربيات دركى كه قبلاً براى فرد ارزش منفى بوده }
\end{aligned}
$$

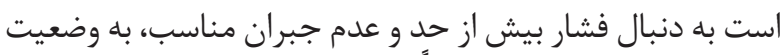

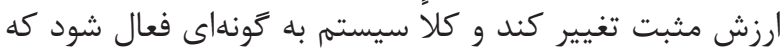

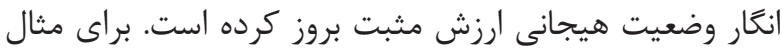

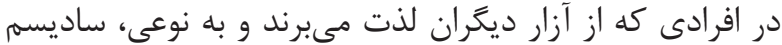

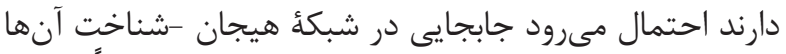

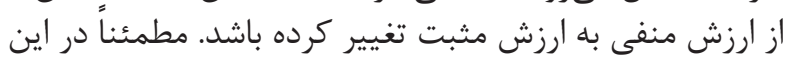

منابع

decision making. Annu Rev Psychol. 2015; 66: 799-823.

11. Schmidt K, Patnaik P, Kensinger EA. Emotion's influence on memory for spatial and temporal context. Cogn Emot. 2011; 25(2): 229-43.

12. Yonelinas AP, Ritchey M. The slow forgetting of emotional episodic memories: an emotional binding account. Trends Cogn Sci. 2015; 19(5): 259-67.

13. Kensinger EA. Negative emotion enhances memory accuracy. Curr Dir Psychol Sci. 2007; 16(4): 213-8.

14. Gozzi A, Jain A, Giovannelli A, Bertollini C, Crestan $\mathrm{V}$, Schwarz AJ, et al. A neural switch for active and passive fear. Neuron. 2010; 67(4): 656-66.

15. Osaka M, Yaoi K, Minamoto T, Osaka N. When do negative and positive emotions modulate working memory performance? Sci Rep. 2013; 3: 1375. doi: 10.1038/srep01375.

16. Okon-Singer H, Hendler T, Pessoa L, Shackman AJ. The neurobiology of emotion-cognition interactions: fundamental questions and strategies for future research. Front Hum Neurosci. 2015; 9: 58. doi: 10.3389/ fnhum.2015.00058.

17. Ghashghaei HT, Hilgetag CC, Barbas H. Sequence of information processing for emotions based on the anatomic dialogue between prefrontal cortex and amygdala. NeuroImage. 2007; 34(3): 905-23.

18. Ray RD, Zald DH. Anatomical insights into the interaction of emotion and cognition in the prefrontal cortex. Neurosci Biobehav Rev. 2012; 36(1): 479-501.

19. Pessoa L. On the relationship between emotion and cognition. Nat Rev Neurosci. 2008; 9(2): 148-58.

20. Ziegler DR, Herman JP. Neurocircuitry of stress 


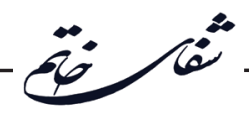

integration: anatomical pathways regulating the hypothalamo-pituitary-adrenocortical axis of the rat. Integr Comp Biol. 2002; 42(3): 541-51.

21. Squire L, Berg D, Bloom FE, du Lac S, Ghosh A, Spitzer NC. Fundamental neuroscience. $4^{\text {th }}$ ed. New York. Academic Press. 2012; P. 1152.

22. Figueiredo HF, Bruestle A, Bodie B, Dolgas CM, Herman JP. The medial prefrontal cortex differentially regulates stress-induced c-fos expression in the forebrain depending on type of stressor. Eur J Neurosci. 2003; 18(8): 2357-64.

23. Shin LM, Rauch SL, Pitman RK. Amygdala, medial prefrontal cortex, and hippocampal function in PTSD. Ann N Y Acad Sci. 2006; 1071: 67-79.

24. Zhuo M. Long-term potentiation in the anterior cingulate cortex and chronic pain. Philos Trans Phys Sci Eng. 2014; 369: 1633. doi: 10.1098/rstb.2013.0146.

25. Silvetti M, Alexander W, Verguts T, Brown JW. From conflict management to reward-based decision making: actors and critics in primate medial frontal cortex. Neurosci Biobehav Rev. 2014; 46: 44-57.

26. Zhuo M. Synaptic and molecular mechanisms of glutamatergic synapses in pain and memory. Sheng Li Xue Bao. 2003; 55(1): 1-8.

27. Ren K, Dubner R. Descending modulation in persistent pain: an update. Pain. 2002; 100(1-2): 1-6.

28. Duquette M, Roy M, Lepore F, Peretz I, Rainville P. Cerebral mechanisms involved in the interaction between pain and emotion. Rev Neurol. 2007; 163(2): 169-79.

29. Sara Susan J, Bouret S. Orienting and reorienting: the locus coeruleus mediates cognition through arousal. Neuron. 2012; 76(1): 130-41.

30. Dreisbach G, Fischer R. The role of affect and reward in the conflict-triggered adjustment of cognitive control. Front Hum Neurosci. 2012; 6: 342. doi: 10.3389/ fnhum.2012.00342.

31. Soloff PH, White R, Omari A, Ramaseshan K, Diwadkar VA. Affective context interferes with brain responses during cognitive processing in borderline personality disorder: fMRI evidence Psychiatry Res. 2015; 233(1): 23-35.

32. Rabinovich MI, Muezzinoglu MK, Strigo I, Bystritsky A. Dynamical principles of emotioncognition interaction: mathematical images of mental disorders. PLoS One. PLoS One. 2010; 5(9): e12547. doi: 10.1371/journal.pone.0012547.

33. Afraimovich V, Young T, Muezzinoglu MK, Rabinovich MI. Nonlinear dynamics of emotioncognition interaction: when emotion does not destroy cognition? Bull Math Biol. 2011; 73(2): 266-84.

34. Jia H, Hu X, Deshpande G. Behavioral relevance of the dynamics of the functional brain connectome. Brain Connect. 2014; 4(9): 741-59.

35. Zhang L, Peng W, Chen J, Hu L. Electrophysiological evidences demonstrating differences in brain functions between nonmusicians and musicians. Sci Rep. 2015; 5. doi:10.1038/srep13796.

36. Schweizer S, Walsh N, Stretton J, Dunn V, Goodyer IM, Dalgleish T. Enhanced emotion regulation capacity, and its neural substrates in those exposed to moderate childhood adversity. Soc Cogn Affect Neurosci. 2016; 11(2): 272-81. 